\title{
Endaural Endoscopic Atticoantrotomy (Retrograde Mastoidectomy) using a Constant Suction Bone-drilling Technique
}

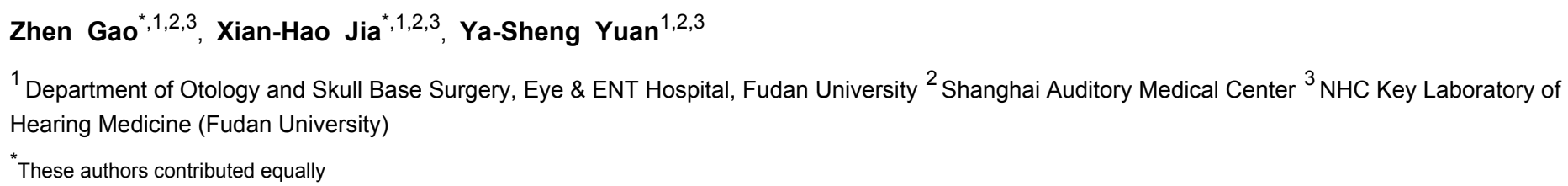

\section{Corresponding Authors}

Zhen Gao

gaozhen_fudan@126.com

Ya-Sheng Yuan

yuanyasheng@163.com

\section{Citation}

Gao, Z., Jia, X.H., Yuan, Y.S. Endaural Endoscopic Atticoantrotomy (Retrograde Mastoidectomy) using a Constant Suction Bone-drilling Technique. J. Vis. Exp. (171), e62450, doi:10.3791/62450 (2021).

\section{Date Published}

May 23, 2021

DOI

$10.3791 / 62450$

URL

\section{Abstract}

Endoscopic middle ear surgery is a widely employed minimally invasive surgical technique to address middle ear and mastoid pathology. Bone drilling is the main technical challenge of endoscopic middle ear surgery. The accompanying video describes the detailed protocol of a constant-suction bone-drilling technique and the procedure of endaural exclusive endoscopic atticoantrotomy (retrograde mastoidectomy) using this technique. The main components of this bone-drilling technique include a soft and flexible suction tube, which is placed into the tympanic cavity to provide constant suction, and a soft sleeve, which is wrapped around the drill shaft to prevent the high-speed rotating shaft from damaging the lens of the endoscope. With these simple modifications, the traditional otological electrodrill can be used for drilling a tiny endaural incision in endoscopic middle ear surgery. Based on this bone-drilling technique, endaural endoscopic atticoantrotomy (retrograde mastoidectomy) can be successfully established for the removal of various amounts of bone, depending on the extent of the lesion. The short-term postoperative outcome seems promising.

jove.com/video/62450

\section{Introduction}

Since the endoscope was introduced as a powerful tool in ear surgery over 20 years ago, endoscopic middle ear surgery or endoscopic middle ear surgery adjunct to conventional microscopic ear surgery has quickly become a widely employed surgical technique to address middle ear and mastoid pathology ${ }^{1,2}$. The main advantages of the endoscope are its panoramic and wide-angle vision. The use of the endoscope reveals the entire structure of the middle ear through the confined external auditory canal. The use of angle endoscopes $\left(30^{\circ}\right.$ and $\left.45^{\circ}\right)$ further facilitates the exposure of the surgical field and allows dissection into the most hidden areas of the middle ear. Multiple studies have demonstrated 
the advantages of the superior visibility of endoscopic procedures over traditional microscopic approaches and confirmed that the major outcomes, such as the recurrence of the lesion and the changes in hearing levels, were not different between the two approaches ${ }^{3,4}$. The advances in technology and surgical instruments are still pushing the boundaries of endoscopic middle ear surgery to manage more disorders of the middle ear and even of the lateral skull base.

However, during transcanal endoscopic ear surgery, as one hand needs to hold the endoscope firmly, all surgical procedures must be performed by the other hand. As a result, surgical techniques of endoscopic ear surgery require some effort, especially the bone drilling technique poses a great technical challenge in endoscopic middle ear surgery. Due to the lack of an efficient endoscopic bone drill technique, some surgeons consider any pathology extending beyond an imaginary line, which bisects the lateral semicircular canal, not to be suitable for an exclusive endoscopic approach. Moreover, when drilling is required, some surgeons would instead switch to a microscope to enable the use of both hands, which may require additional postauricular or endaural incisions to improve access.

To address this problem, a constant-suction bonedrilling technique has been developed in these authors' department for endaural exclusive endoscopic atticoantrotomy (retrograde mastoidectomy). From April 2020 to August 2020, 11 cases of endaural exclusive endoscopic atticoantrotomy have been successfully performed with this technique in the department. The purpose of this video is to describe the detailed procedure of this constant-suction bone-drilling technique and endaural exclusive endoscopic atticoantrotomy using this technique.

\section{Protocol}

NOTE: This study was approved and performed according to the guidelines of the ethical committee of Fudan University.

\section{Anesthesia and surgical field preparation}

1. Induce intravenous anesthesia and perform orotracheal intubation or use a laryngeal mask.

2. Place the patient in a supine position with the head rotated to the contralateral side.

3. Sterilize the auricle and the external ear canal (EAC) with a povidone iodine solution; drape the surgical field with a blanket sparing the EAC.

4. Check the optical clarity of the endoscope and adjust its white balance.

5. Introduce the $0^{\circ}, 3 \mathrm{~mm}$ diameter, and $15 \mathrm{~cm}$ length endoscope into the EAC. Remove the cerumen from the EAC with microscopic forceps and sterilize the EAC with the povidone iodine solution again.

\section{Establishment of a minimal invasive endaural approach}

1. Administer local anesthesia to the EAC by injecting $1 \%$ lidocaine with adrenaline $(1: 100,000)$ at 7 and 11 -o'clock positions.

2. Under the endoscope, make a circumferential incision with a round knife from 2 to 6-o'clock position, counterclockwise (right ear), approximately $8 \mathrm{~mm}$ from the tympanic membrane.

NOTE: Use povidone iodine to clean the lens of the endoscope. 
3. Raise the tympanomeatal flap with a $1 \mathrm{~mm}$ diameter suction tube and a small piece of gauze soaked with adrenaline $(1: 1,000)$.

4. Under direct vision, make an intercartilaginous incision with a scalpel at the 12-o'clock position, from the previously made circumferential incision towards the meatus and through the stretched intercartilaginous incisura.

5. Make another radial incision with the scalpel at the 6o'clock position, from the previously made circumferential incision towards the meatus.

6. Raise the flap enclosed by the medial circumferential incision and the two radial incisions outward with a curette. Check the exposure of the lateral wall of the attic and the posterior wall of the EAC.

NOTE: The edge of the incision can be subjected to bipolar coagulation so that it shrinks, providing better exposure.

7. Place a self-retaining retractor to widen the meatus of the EAC and hold the lateral-based skin flap in place.

\section{Bone drilling and lesion removal}

1. Tailor the tube of a disposable venous infusion needle to make a soft suction tube, which will be used in the bonedrilling stage. Make several side holes at its distal end with scissors (Figure 1).

2. Cut a small section of the tube of a disposable infusion set to make an electrodrill shaft sleeve that will protect the lens of the endoscope during bone drilling. Fix the sleeve to the drill handle with a sterile transparent sticker (Figure 1).
3. Put the distal end of the soft suction tube (with the side holes) into the tympanic cavity, and connect the other end of the tube to a vacuum aspirator.

4. Execute bone drilling in an inside-out direction starting from the posterior part of the scutum.

NOTE: Here, a $1.8 \mathrm{~mm}$ diamond burr is used in the beginning to avoid tearing the tympanomeatal flap. After removing the posterior part of the scutum, lesions around the incudostapedial joint can be fully exposed. Drilling of the posterior lateral attic wall can be expanded If the lesion cannot be removed entirely.

5. Instruct the assistant to irrigate the meatus of the EAC with saline continuously.

NOTE: If necessary, additional clearance of bone sludge can also be done intermittently with the surgical suction tube.

6. Remove the anterior part of the lateral attic wall by bone drilling if the lesion extends anteriorly.

NOTE: The malleus head can be removed to facilitate the lesion removal.

7. Perform an extended atticoantrotomy if the lesion extends further into the antrum.

NOTE: The whole attic and antrum can be opened to facilitate lesion removal. Sometimes, the direct approach can be applied to the retrotympanum to remove the lesion with minimal bone-drilling ${ }^{5}$. In such an approach, the surgeon stands opposite to the affected ear and uses a $45^{\circ}$ endoscope. The operating table should be tilted $\sim 30^{\circ}$ toward the surgeon to facilitate the introduction of the endoscope and the instruments. After lesion removal, lens fogging can be observed in some patients when the constant suction is stopped. This phenomenon will disappear if the tympanic orifice of the eustachian tube 
is blocked by a small piece of gauze. Of note, such a phenomenon could be used to assess eustachian tube function (fogging is a sign of good function). To observe this phenomenon, the balloon should not be too tight when endotracheal intubation is established.

\section{Reconstruction and closure}

1. Check the integrity and mobility of the ossicular chain after lesion removal. Correct ossicular abnormalities with various mature surgical techniques ${ }^{6}$.

2. Harvest a large piece of tragus cartilage with perichondrium on both sides.

3. On one side, detach the perichondrium from the cartilage with a round knife, leaving the perichondrium on the other side to form a cartilage-perichondrium graft. Tailor the cartilage-perichondrium graft for reconstruction.

4. Use the cartilage-perichondrium graft and the perichondrium to reconstruct the lateral wall of the attic and the tympanic membrane.

NOTE: If an extended atticoantrotomy is performed, part of the cartilage-perichondrium graft can also be used to reconstruct the posterior wall of the EAC.

5. Tailor and reposition the tympanomeatal flap and the lateral-based skin flap; pack the EAC with a gelatin sponge.

6. Suture the intercartilaginous incision and pack it with gauze.

\section{Postoperative management}

1. Administer intravenous antibiotics for 2 days postoperatively during hospitalization.
2. Remove the dressing 2 days after the surgery, just before discharge.

3. Apply oral antibiotics and ciprofloxacin drops for 2 weeks after discharge.

4. Remove the stitches and the remaining gelatin sponge at the first visit, usually 7 to 10 days after discharge.

5. Organize future follow-ups according to the pathology and type of surgery.

\section{Representative Results}

From April 2020 to August 2020, the senior surgeon performed endaural exclusive endoscopic atticoantrotomy on 11 patients using the above-mentioned constant-suction bone-drilling technique. The patients' characteristics are described in Table 1. There were 10 men (90.9\%) and 1 woman $(9.1 \%)$. The patients' age ranged from 9 to 63 years, with a mean age of 41 years. Most of these cases were cholesteatoma (8 cases), followed by chronic suppurative otitis media ( 3 cases). Of the 8 patients with cholesteatoma, the most common sites of involvement were attic $(n=8,100$ $\%)$, followed by antrum ( $n=7,87.5 \%)$ and tympanic cavity $(n=3,37.5 \%)$.

Intraoperative ossicular chain reconstruction was performed in 8 cases $(72.7 \%)$ using methods including titanium partial ossicular replacement prostheses (4 cases) and titanium total ossicular replacement prostheses (4 cases). The results of short-term follow-up seem promising. Until the revisit at 3 months after the surgery, no sign of recurrence was noticed by clinical examination. However, the results of long-term follow-up are still unclear, which indicates further investigation is still needed. 
With respect to the hearing outcome, a better postoperative air-bone gap (ABG) was observed in 5 patients, no significant change was observed in $A B G$ in 5 patients, and a worse postoperative ABG was noted in 1 patient. The postoperative facial nerve function was normal in all the patients. No other complications were noticed postoperatively. The photographs of typical cases are shown in Figure 2.
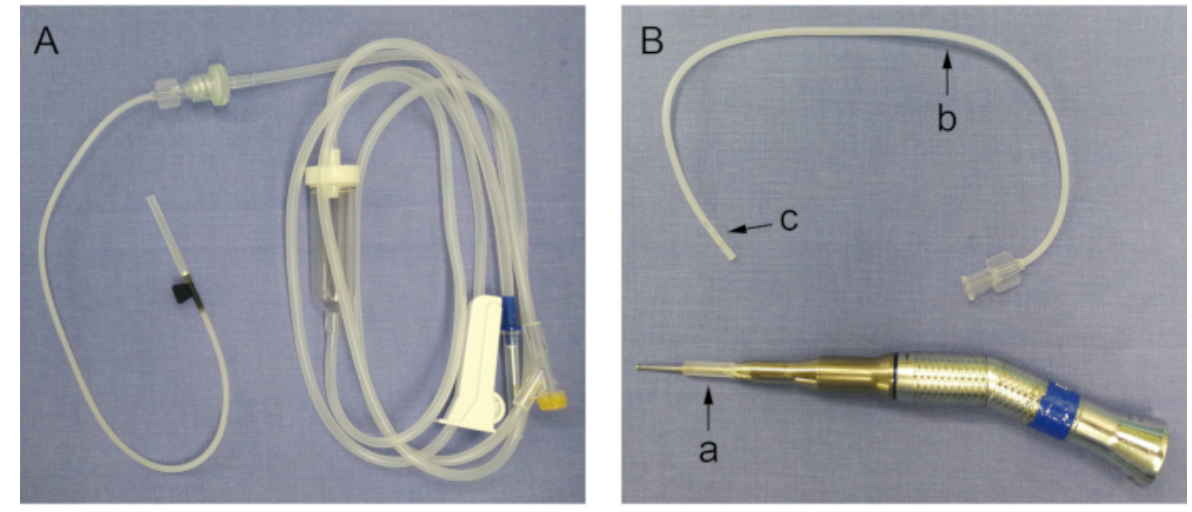

Figure 1: Tailoring of a disposable infusion set and venous infusion needle and modification of electrodrill

handpiece. (A) The disposable infusion set and venous infusion needle before tailoring. (B) The components cut out of the disposable infusion set and venous infusion needle: (a) electrodrill shaft sleeve, which is fixed on the handpiece with a sterile transparent sticker, (b) the soft suction tube, which will be inserted into the tympanic cavity, (c) side holes on the suction tube. Please click here to view a larger version of this figure. 

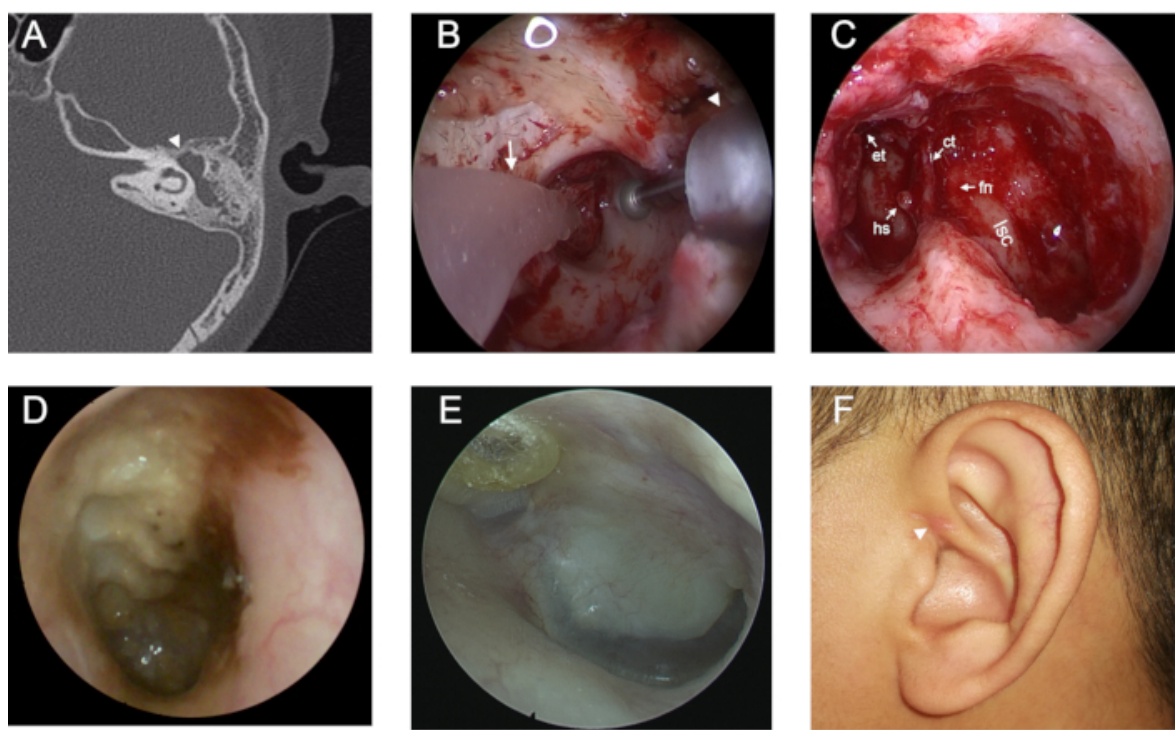

Figure 2: Typical cases. (A) Axial CT image of one patient shows cholesteatoma (arrowhead) in the attic and antrum. Note that the extent of the lesion goes beyond the imaginary line that bisects the lateral semicircular canal. (B) Establishment of constant-suction bone-drilling technique. Arrow indicates the soft suction tube; arrowhead indicates the electrodrill shaft sleeve. (C) The operative cavity of the same patient in (A) after completion of the endaural endoscopic atticoantrotomy. (D) Preoperative endoscopic image of the tympanic membrane. (E) Endoscopic image of the tympanic membrane of the same patient in (D) at 6 months after the operation. (F) One month after the surgery, the endaural incision (indicated by the arrowhead) is almost invisible. Abbreviations: $\mathrm{CT}=$ computed tomography; et = eustachian tube; $\mathrm{hs}=$ head of stape; ct = chorda tympani; $\mathrm{fn}$ = facial nerve; Isc = lateral semicircular canal. Please click here to view a larger version of this figure. 


\begin{tabular}{|c|c|c|c|c|c|c|c|c|}
\hline No & Sex & age & side & lesion & extent & $\begin{array}{l}\text { Ossicular chain } \\
\text { reconstruction }\end{array}$ & ABG preoperative & $\begin{array}{c}\text { ABG } 3 \\
\text { months } \\
\text { later }\end{array}$ \\
\hline 1 & $M$ & 33 & $\mathrm{R}$ & cholesteatoma & $\begin{array}{c}\text { tympanic } \\
\text { cavity,attic,antrum }\end{array}$ & TORP & 34 & 20 \\
\hline 2 & M & 47 & $\mathrm{~L}$ & cholesteatoma & attic, antrum & TORP & 32 & 22 \\
\hline 3 & M & 28 & $\mathrm{~L}$ & cholesteatoma & $\begin{array}{c}\text { tympanic } \\
\text { cavity,attic,antrum }\end{array}$ & TORP & 38 & 43 \\
\hline 4 & $M$ & 59 & $\mathrm{~L}$ & cholesteatoma & $\begin{array}{c}\text { tympanic } \\
\text { cavity,attic,antrum }\end{array}$ & PORP & 27 & 22 \\
\hline 5 & M & 62 & $\mathrm{R}$ & CSOM & $\begin{array}{c}\text { tympanic } \\
\text { cavity,attic,antrum }\end{array}$ & not reconstructed & $\begin{array}{l}\text { profound } \\
\text { deafness }\end{array}$ & $\begin{array}{l}\text { profound } \\
\text { deafness }\end{array}$ \\
\hline 6 & $\mathrm{~F}$ & 53 & $\mathrm{~L}$ & CSOM & $\begin{array}{c}\text { tympanic } \\
\text { cavity,attic,antrum }\end{array}$ & not reconstructed & 25 & 15 \\
\hline 7 & M & 33 & L & cholesteatoma & attic,antrum & PORP & 43 & 23 \\
\hline 8 & $M$ & 9 & $\mathrm{~L}$ & cholesteatoma & attic, antrum & PORP & 20 & 21 \\
\hline 9 & $M$ & 41 & $\mathrm{~L}$ & cholesteatoma & attic & TORP & 10 & 34 \\
\hline 10 & M & 28 & $\mathrm{~L}$ & cholesteatoma & attic, antrum & not reconstructed & 10 & 13 \\
\hline 11 & $M$ & 63 & $\mathrm{R}$ & CSOM & $\begin{array}{c}\text { tympanic } \\
\text { cavity,attic,antrum }\end{array}$ & PORP & 45 & 23 \\
\hline
\end{tabular}

Table 1: Characteristics and short-term outcomes of patients who underwent endaural endoscopic atticoantrotomy with the constant-suction bone-drilling technique. Abbreviations: $M=$ male; $F=$ female; $R=$ right; $L=$ left; $C S O M=$ chronic suppurative otitis media; TORP = total ossicular replacement prostheses; $P O R P=$ partial ossicular replacement prostheses; $A B G=$ air bone gap.

\section{Discussion}

Since the introduction of the endoscope in middle ear surgery in the $1990 \mathrm{~s}^{7,8}$, endoscopic ear surgery has gained great popularity around the world. This is partially because the endoscope can provide high-quality images and help visualize poorly seen structures such as the hypotympanum and sinus tympani. Moreover, the EAC, which is a much more direct and natural approach than the retroauricular approach to the middle ear, confers the advantages of less trauma and quick recovery. The accompanying video provides a detailed protocol of endaural exclusive endoscopic atticoantrotomy using a constant-suction bone- 
drilling technique adopted at this hospital. The procedure mentioned above is an endoscopic modification of the insideout transmeatal mastoidectomy (retrograde mastoidectomy), which has already been developed in the microscope era ${ }^{9,10}$. In the current technique, we retain the advantages of the primary microscopic inside-out technique, such as a direct and natural approach, less bone removal, and following the lesion along the direction of its growth. The use of the endoscope eliminates the original disadvantages such as inadequate exposure and limited visualization.

The surgical technique mentioned above provides an endoscopic solution to various middle ear lesions, especially different degrees of cholesteatoma. Bone drilling can be gradually expanded safely by following the pathology from the tympanic cavity to the attic and the antrum. Cholesteatoma in the tympanic cavity is first recognized after the elevation of the tympanomeatal flap. If the lesion extends superiorly through the tympanic isthmus to the attic, the posterior part of the scutum can be removed first. If the cholesteatoma cannot be removed entirely, drilling could be expanded posteriorly and superiorly toward the aditus and the posterior part of the attic. If the lesion further extends anteriorly, the atticotomy could be expanded by drilling the lateral attic wall anteriorly to expose the malleus head. In case of further posterior extension of the cholesteatoma into the antrum, drilling is continued posteriorly along the tegmen tympani and tegmen antri, with a typical atticoantrotomy being performed. In summary, the extent of endaural endoscopic atticoantrotomy (retrograde mastoidectomy) is flexible and based on the requirement.

In the authors' department, this technique is mainly used in selected patients with cholesteatoma confined to the attic and the antrum, especially in the ones with sclerotic mastoid. This surgical technique can also be used to enlarge the posterior bony part of the EAC, which is quite helpful for some patients with EAC stenosis. Currently, bone drilling is a key technical challenge of endoscopic middle ear surgery. This is mainly due to the narrow feature of the EAC, which makes it almost impossible to insert multiple instruments simultaneously. In addition, the surgeon must hold the endoscope constantly in one hand, making drilling while irrigating quite difficult. When drilling is required, some surgeons advocate switching to a microscope to enable the use of both hands. Several drilling techniques have also been developed for endoscopic middle ear surgery, such as drilling in a watered field and the "chopstick" technique ${ }^{5,11}$.

In this video, we show methods to achieve bone drilling while irrigating and suctioning at the same time. This technique has several advantages. First, it is a simple but efficient method. After establishing a tiny endaural incision and simply modifying the electrodrill handpiece, the traditional otological electrodrill can be as effective as the specially designed microdrill or an ultrasonic bone curette for bone removal in endoscopic middle ear surgery. Second, the suction tube placed in the EAC is soft and flexible, providing great agility and maneuverability that facilitates operation in tight spaces. Finally, the soft sleeve around the drill shaft is also quite useful. It can prevent the high-speed rotating shaft from damaging the lens of the endoscope. Thus, the drilling technique mentioned in this paper is practical and efficient and can be successfully adopted in endoscopic middle ear surgery.

\section{Disclosures}

The authors declare that there are no conflicts of interest regarding the publication of this article.

\section{Acknowledgments}


This study was supported by Youth Fund of National Natural

Science Foundation of China (Grant No. 81400460), Youth

Fund of National Natural Science Foundation of China (Grant No. 81900932), and Clinical Research Plan of SHDC (Grant No. SHDC2020CR1049B).

\section{References}

1. Yong, M., Mijovic T., Lea J. Endoscopic ear surgery in Canada: a cross-sectional study. Journal of Otolaryngology-Head \& Neck Surgery. 45, 4 (2016).

2. Kapadiya, M., Tarabichi, M. An overview of endoscopic ear surgery in 2018. Laryngoscope Investigative Otolaryngology. 4 (3), 365-373 (2019).

3. Tseng, C. C., Lai, M. T., Wu, C. C., Yuan, S. P., Ding, Y. F. Endoscopic transcanal myringoplasty for anterior perforations of the tympanic membrane. JAMA Otolaryngology-Head \& Neck Surgery. 142, 1088-1093 (2016).

4. Huang, Y. B., Hu, L. L., Ren, D. D., Han, Z. Myringoplasty with an ultrathin cartilage-perichondrium complex graft versus temporalis fascia graft: a propensity score-matched analysis. Otolaryngology-Head and Neck Surgery. 194599820965940 (2020).

5. Presutti, L., Marchioni, D. Endoscopic ear surgery: principles, indications, and techniques. Thieme. (2015).

6. Slater, P. W., Rizer, F. M., Schuring, A. G., Lippy, W. H. Practical use of total and partial ossicular replacement prostheses in ossiculoplasty. Laryngoscope. 107 (9), 1193-1198 (1997).

7. Thomassin, J. M. et al. Endoscopic ear surgery. Annales d' Oto-laryngologie et de Chirurgie Cervico Faciale. 107 (8), 564-570 (1990).
8. Pollak, N. Endoscopic and minimally-invasive ear surgery: A path to better outcomes. World Journal of Otorhinolaryngology-Head and Neck Surgery. 3 (3), 129-135 (2017).

9. Dornhoffer, J. L. Retrograde mastoidectomy. Otolaryngologic Clinics of North America. 39 (6), 1115-1127 (2006).

10. Hatano, M., Ito, M., Yoshizaki, T. Retrograde mastoidectomy on demand with soft-wall reconstruction in pediatric cholesteatoma. Acta Oto-laryngologica. 130 (10), 1113-1118 (2010).

11. Vaughan, C., Jufas N., Patel, N. P., Saxby, A. J., Kong, J. H. K. The "Chopstick Drilling Technique": A novel solution to drilling bone in endoscopic ear surgery. Clinical Otolaryngology. 44 (6), 1231-1233 (2019). 